\title{
Complications Following Urethral Reconstructive Surgery: A Six Year Experience
}

\author{
Neema Navai, Bradley A. Erickson, Lee C. Zhao, Onisuru T. Okotie, Chris M. Gonzalez \\ Department of Urology, Feinberg School of Medicine, Northwestern University, Chicago, Illinois, \\ USA
}

\begin{abstract}
Purpose: We present a single institutional experience over 6 years of intra and postoperative complications following urethral reconstructive surgery, and the impact of these complications on overall results.

Materials and Methods: From June 2000 through May 2006, 153 consecutive urethral reconstructive procedures were performed on 128 patients by one surgeon $(\mathrm{CMG})$. Complication rates were determined, and subgroups were categorized based on stricture etiology, patient age, length of stricture, location of stricture, type of repair, and presence of various co-morbid conditions.

Results: Overall, 23 of 153 cases (15\%) had an intra or postoperative complication with a mean follow-up time of 28.3 months (range 3 to 74$)$. The most common complications were related to infection $(\mathrm{n}=9)$ ). Other complications included repair breakdown $(n=4)$, bleeding $(n=4)$, fistulae $(n=3)$, thromboembolic $(n=2)$, positioning-related $(n=2)$, and Foley catheter malfunction $(n=1)$. Complication rates for anastomotic and substitution urethroplasty were $9.1 \%(4 / 44)$ and $17 \%(19 / 109)$, respectively. The number of patients with at least one year of follow-up who had a complication and eventual stricture recurrence was $20 \%(4 / 20)$, while only $7.4 \%$ (7/95) of those who did not have a complication recurred $(\mathrm{p}=0.08)$.

Conclusions: Complications following reconstructive surgery for urethral stricture disease were mostly related to infection or repair breakdown in the immediate postoperative period. It does not appear that an intra or postoperative complication following urethral reconstructive surgery impacts the chance of eventual stricture recurrence at intermediate follow-up.
\end{abstract}

Key words: urethral stricture; urethroplasty; complications; recurrence

Int Braz J Urol. 2008; 34: 594-601

\section{INTRODUCTION}

Reconstructive urethral surgery has been shown to be an effective treatment for urethral stricture disease with durable results (1-4). However, there are few major studies specifically analyzing complications following or during urethral reconstruction surgery. In a series of 60 patients, Al-Qudah et al. reported complication rates as high as $48 \%$, though most of these events were classified as minor (5). In one of the largest reconstructive series to date,
Andrich et al. reported that complications following reconstructive surgery have ranged from as low as $7 \%$ for excision and primary anastomosis to as high as $33 \%$ following substitution urethroplasty (1).

We assessed our experience with 153 consecutive urethral reconstructive cases over a 6-year period by a single surgeon within the same institution. A retrospective review of all urethral reconstructions, complications and recurrences over a 6-year period was performed. Our purpose was to determine the overall incidence and specific type of complications 
that can occur during or after a variety of urethral reconstructive procedures, along with the impact these associated complications may have on disease recurrence. Specific patient demographics, co-morbid conditions, stricture characteristics, and reconstructive techniques were analyzed for complication rates.

\section{MATERIALS AND METHODS}

We evaluated patients who underwent urethral reconstructive surgery by a single surgeon (CMG) from June 2000 through May 2006. Follow-up was through July 2006 to ensure at least 60 days of follow-up per patient. All research activities met the approval of the Institutional Review Board. A total of 128 patients underwent 153 consecutive urethral reconstructive procedures during this period. Patients who underwent two-staged repairs account for the discrepancy between the number of reconstructive procedures and the number of patients. Patient characteristics, type of surgical procedure, stricture location, stricture length, stricture recurrence, patient age, complications, and presence of co-morbid conditions were prospectively entered into a database. Additionally, all inpatient and outpatient records were retrospectively analyzed to confirm the findings. The type of reconstructive procedure was determined by the surgeon after preoperative radiographic and endoscopic evaluation of the stricture, and ultimately after surgical evaluation of the stricture extent. Procedures were either done in dorsal lithotomy, supine or in exaggerated lithotomy. To prevent neurologic complications in patients placed in the exaggerated lithotomy position we utilized Yellofins ${ }^{\circledR}$ stirrups (Allen Medical Systems), and a $1 \mathrm{~L}$ saline bag wrapped in a blue towel to support the lumbar spine. Following urethral reconstruction a Foley catheter was routinely maintained indwelling for 3 weeks in those patients undergoing substitution urethroplasty with a graft or flap, and for 2 weeks in those men undergoing primary anastomosis, including those undergoing repair for urethral erosions. We made no distinction between perceived minor or major complications. Complications were classified as infection-related, bleeding, thromboembolic (deep vein thrombosis and pulmonary embolism), positioning-related, surgical repair breakdown, fistula formation, and Foley malfunction. Stricture recurrence rates were also evaluated, which we defined as the endoscopic identification of the urethral lumen at the repair site of less then $18 \mathrm{~F}$ in diameter.

All postoperative patients were screened for recurrence through assessment of subjective voiding symptoms, ultrasound post-void residual, and urine culture. Routine invasive monitoring with cystoscopy and retrograde urethrogram was not performed. Sexual dysfunction was not included as a complication in our study. Specific co-morbid conditions evaluated in this study included neurogenic bladder, prior renal or pancreas transplantation (enteric or bladder drained), penile or urethral lichen sclerosis including balanitis xerotica obliterans, previous hypospadias repair, and diabetes mellitus. Additionally, the etiology of stricture disease was recorded if it could be determined from the patient's initial history or previous medical record. Those strictures with no known etiology were classified as idiopathic. All patients received intravenous ampicillin (clindamycin or vancomycin if penicillin allergic) and gentamicin (ciprofloxacin if renal failure or insufficiency) peri-operatively, and all urine cultures were confirmed negative prior to surgery. For deep venous thrombosis prevention, compression stockings and sequential compression devices were used for patients in the supine and low lithotomy position, with only compression stockings used for those in the exaggerated lithotomy position.

\section{RESULTS}

Table-1 shows the distribution of types of repair and their associated complication rates. Mean patient age was 41.5 years (15 to 79 SD 14.5 years), with a mean follow-up of 28.3 months (SD 19.1 months). The average stricture length was $5.5 \mathrm{~cm}$ (1 to $22 \mathrm{SD} 3.7 \mathrm{~cm}$ ). Table-2 shows the distribution of stricture length by type of repair. Grafts and flaps used were as follows; 74 buccal grafts, 4 posterior auricular grafts, 2 scrotal skin grafts, 1 abdominal wall skin graft, 4 penile skin grafts, 5 circular fasciocutaneous flaps, 3 penile skin flaps, and 65 cases were done without the use of flap or graft. The total number of grafts 
Table 1 - Complication rate by type of urethral stricture repair.

\begin{tabular}{lcc}
\hline Type of Repair & Total Procedures & $\begin{array}{c}\text { Procedures with } \\
\text { Complications (\%) }\end{array}$ \\
\hline Anterior urethral excision and primary anastomosis (EPA) & 33 & $2(6)$ \\
Posterior urethral EPA & 8 & $1(13)$ \\
Diverticulum EPA & 3 & $1(33)$ \\
Bulbar urethral repair with buccal mucosa graft & 38 & $5(13)$ \\
Two-staged repair & 36 & $7(19)$ \\
Penile one-staged with graft or flap & 23 & $5(22)$ \\
Distal one-staged & 6 & $1(17)$ \\
Panurethral & 3 & $1(33)$ \\
Fistula repair & 3 & 0 \\
\hline
\end{tabular}

$E P A=$ excision and primary anastomosis.

used in all cases exceeded the number of procedures secondary to the use of multiple grafts and /or flaps for long segment stricture repair.

Of the 153 cases, 23 cases had complications $(15 \%)$. Two of these cases had multiple complications for a total of 25 recorded adverse events. There were no perioperative deaths. The most common type of complication was infection-related. Five of these were wound complications, including a scrotal abscess and a necrotizing glans penis infection in a patient with insulin dependent diabetes. Three of the infection-related complications were asymptomatic urinary tract infections, and there was one incident of postoperative urosepsis in a patient with a neurogenic bladder. Bleeding complications occurred in 4 patients, including one episode of excessive intraoperative bleeding requiring transfusion. Of the 3 complications that did not receive transfusions, 2 patients a developed perineal hematoma postoperatively and one had an episode of excessive bleeding from the buccal mucosa harvest site, which was managed conservatively with epinephrine-soaked packing. Four patients that underwent urethral erosion repairs secondary to chronic indwelling Foley catheter use suffered partial repair breakdown distally with an additional 3 patients developing a postoperative urethrocutaneous fistula; 2 of these spontaneously healed with Foley catheter drainage after one week. There were 2 thromboembolic events, including one patient with a deep vein thrombosis and another with a pulmonary embolism, which were related to a previously undiagnosed prothrombin gene mutation.

Table 2 - Stricture length by type of surgical repair.

\begin{tabular}{lcccc}
\hline Procedure Code & Total & $\begin{array}{c}\text { Minimum Length } \\
(\mathrm{cm})\end{array}$ & $\begin{array}{c}\text { Maximum Length } \\
(\mathrm{cm})\end{array}$ & $\begin{array}{c}\text { Average Length } \\
(\mathrm{cm})\end{array}$ \\
\hline Excision and primary anastomosis & 44 & 1.5 & 5 & 2.8 \\
Two-staged & 36 & 4 & 14 & 7.9 \\
Distal one-staged & 6 & 2 & 4 & 2.7 \\
Penile one-staged & 23 & 2 & 17 & 6.9 \\
Bulbar w/ graft or flap & 38 & 3 & 7 & 5.0 \\
Long segment & 3 & 11 & 22 & 18 \\
Fistula repair & 3 & 1 & 12 & 5.3 \\
\hline
\end{tabular}


Of the 89 patients who underwent reconstruction in the high lithotomy position, only $2 / 89(2.2 \%)$ had a complication related to positioning. In one of the first cases completed in this series, a young man suffered a severe femoral neuropathy most likely as a result of an operative time in the exaggerated lithotomy position in excess of five hours. The other patient had a temporary lateral foot paresthesia, which resolved spontaneously after three days. There were no positioning-related complications in patients who underwent repair in the dorsal lithotomy $(n=15)$ or supine positions $(n=49)$.

Table-3 shows the distribution of overall complications for the anastomotic and substitution urethral reconstructive cases. There was a complication rate of $9 \%(4 / 44)$ associated with anastomotic urethroplasty which included bulbar urethroplasty, membranous urethroplasty, and diverticulum repair when primary anastomosis was feasible. Substitution urethroplasty cases were found to have a complication rate of $17 \%$ (19/109). Chi-squared analysis was performed on these data, and although there was a trend towards more complications in the substitution urethroplasty subset, statistical significance was not achieved $(p=$ 0.19). Table-4 shows complication data, which was stratified by patient characteristics, including age, stricture location, stricture etiology, and the presence of co-morbid conditions. These factors did not appear to impact upon the overall complication rate; however, given the limited number of complications within each subgroup, statistical analysis could not be made in most cases. To assess if complications during or following surgery impacted upon eventual stricture recurrence, we evaluated all men with at least one year of follow-up in this series. These men experienced an overall complication rate of $17 \%$ (20/115 cases), with an overall recurrence rate of $10.9 \%(11 / 101 \mathrm{men})$. The mean time to stricture recurrence for these men was 130 days (17 to 287 , SD 134 days) with an average overall follow-up time of 35 months (12 to 74, SD 17 months). Strictures repaired with excision and primary anastomosis had a $7.7 \%(2 / 26)$ recurrence rate, whereas those repaired with substitution urethroplasty had a $10.1 \%(9 / 89)$ recurrence rate. Overall, 20\% (4/20) of patients that suffered postoperative complications of any type had stricture recurrence, while only $7.4 \%$ (7/95) of those who did not have a complication recurred. Chi-squared analysis of these two groups showed a nonsignificant trend towards more recurrences in patients with complications $(p=0.08)$.

\section{COMMENTS}

We sought to elucidate and describe complications following 153 consecutive reconstructive pro-

Table 3 - Types of complications for anastomotic and substitution repairs.

\begin{tabular}{lccc}
\hline Complication Code & Anastomotic & Substitution & Total \\
\hline None & 40 & 90 & 130 \\
Infectious & 2 & 7 & 9 \\
Positioning-related & 1 & 1 & 2 \\
Bleeding & 1 & 3 & 4 \\
Thromboembolic & 0 & 2 & 2 \\
Fistula & 0 & 3 & 3 \\
Breakdown & 0 & 4 & 4 \\
Foley catheter malfunction & 0 & 1 & 1 \\
Total cases with complications & 4 & 19 & 23 \\
Total complications & 4 & 21 & 25 \\
Total cases & 44 & 109 & 153 \\
Complication rate & $9 \%(4 / 44)$ & $17 \%(19 / 109)$ & $\mathrm{p}=0.19$ \\
\hline
\end{tabular}


Table 4 - Complication rate by age, etiology of stricture, co-morbid disease, and stricture location.

\begin{tabular}{|c|c|c|}
\hline Age Group (years) & Total Patients & Complications (\%) \\
\hline$<20$ & 5 & $1(20)$ \\
\hline $21-30$ & 32 & $4(13)$ \\
\hline $31-40$ & 40 & $6(15)$ \\
\hline $41-50$ & 37 & $3(8)$ \\
\hline $51-60$ & 20 & $4(20)$ \\
\hline$>60$ & 19 & $5(26)$ \\
\hline \multicolumn{3}{|l|}{ Etiology of stricture } \\
\hline Idiopathic & 73 & $9(12)$ \\
\hline Infectious & 6 & $4(67)$ \\
\hline Erosion & 7 & $1(14)$ \\
\hline Instrumentation & 15 & $4(27)$ \\
\hline Lichen sclerosis & 9 & $1(11)$ \\
\hline Hypospadias & 20 & $2(10)$ \\
\hline Prior urethroplasty & 9 & $2(22)$ \\
\hline Trauma & 13 & 0 \\
\hline Other & 1 & 0 \\
\hline \multicolumn{3}{|l|}{ Co-morbid disease } \\
\hline None & 95 & $12(13)$ \\
\hline Neurogenic bladder & 12 & $2(17)$ \\
\hline Transplant & 8 & $4(50)$ \\
\hline Lichen sclerosis / BXO & 8 & 0 \\
\hline Hypospadias & 24 & $4(17)$ \\
\hline Both transplant and neurogenic bladder & 1 & 0 \\
\hline Diabetes mellitus & 5 & $1(20)$ \\
\hline \multicolumn{3}{|l|}{ Stricture location } \\
\hline Fossa navicularis & 8 & $1(13)$ \\
\hline Penile & 58 & $12(21)$ \\
\hline Bulbar & 79 & $9(11)$ \\
\hline Membranous & 5 & 0 \\
\hline Panurethral & 3 & $1(33)$ \\
\hline
\end{tabular}

$B X O=$ balanitis xerotica obliterans.

cedures for urethral stricture disease and the impact of these events on disease recurrence. With recent studies lending credence to broadening the use of formal urethral reconstruction $(6,7)$ we believe a more complete understanding of complications following the multitude of available procedures for stricture disease is necessary for appropriate patient counseling.
Additionally, for the purposes of this study, we defined a complication as any adverse event or inadvertent deviation from the standard of care either during or after urethral reconstructive surgery. Our intention was to include all complications, however minor, without discrimination. Thus, we did not differentiate between perceived major and minor complications. 
Postoperative sexual dysfunction was not included in this analysis as we have previously reported on these data from this same series (8).

The complication rate for anastomotic and substitution urethroplasty in this series was $9 \%$ and $17 \%$, respectively. A comparison of these two groups did not reach a statistical difference $(p=0.19)$; however, a similar trend of complications following primary anastomosis and substitution urethroplasty has been previously reported at $7 \%$ and $33 \%$, respectively (1). It is unclear why there were more complications in the substitution urethroplasty group as compared to the anastomotic group despite grouping posterior urethroplasty cases into the primary anastomosis group; however, contributing factors such as increased length of stricture and the need for harvest and interposition of graft tissue in the substitution urethroplasty group may factor into these findings. We also analyzed the number of complications related to one-stage $6 / 29(21 \%)$ versus two-stage $7 / 36(19 \%)$ procedures involving the penile urethra and fossa navicularis. Despite the fact that most of the strictures requiring two-stage procedures were more complex in etiology (i.e. hypospadias failure, failed prior reconstruction), no significant difference between the two groups was found $(\mathrm{p}=0.90)$.

The high lithotomy position for urethral reconstructive surgery has been associated with complication rates between 10-16\% (9-12). In our study, complications related to the high lithotomy position were limited and only occurred in two $(2.2 \%)$ of the 89 cases performed. Major factors responsible for the limited number of positioning-related complications in this series included the self imposed limit of having the patient in exaggerated lithotomy for less than five hours, the use of specialized stirrups, lower back support, and high patient volume. Positioning-related complications in the supine and dorsal lithotomy position did not occur in this series.

The majority of complications in our series were related to infection. Despite the administration of peri-operative antibiotics, and a negative preoperative urine culture, wound-related infections accounted for $5 / 153(3.2 \%)$ cases. Despite the relative frequency of these occurrences in this series, these data are comparable to wound infection rates reported for similar procedures including perineal prostatectomy (1.6\%), circumcision (1.3\%) and hydrocele repair (4\%) (13$15)$.

Finally, we attempted to evaluate whether a complication following urethral reconstructive surgery increased the chance of eventual stricture recurrence. In order to avoid underestimation of recurrence, all men with less then one year of followup in this series were excluded. A recurrence rate of $10.9 \%$ was found in these men, which is similar to that of previously reported data $(16,17)$. Although $20 \%$ of men with a complication eventually experienced a recurrence at a mean follow-up time of just less than three years, a statistically significant difference could not be found between those without a complication and those with an eventual recurrence. Long-term data are needed to confirm these findings at intermediate follow-up.

One of the major limitations of this study was the inability to perform statistical analysis on some of the subgroups due to the limited number of complications. We pooled similar subgroup for analysis when applicable; however, because of the relatively low number of events, meaningful statistical analysis could not be performed in some subgroups. Multi-institutional studies would be helpful in providing the appropriate statistical power necessary to determine if some of these potential co-morbidities may predispose a patient to a complication, thus allowing the employment of appropriate preventative measures. Nonetheless, to the best of our knowledge, this is one of the largest series of complications reported in consecutive patients undergoing urethral reconstruction surgery for stricture disease.

\section{CONCLUSION}

Complications following reconstructive surgery for urethral stricture disease were minor and mostly related to infection and repair breakdown in the postoperative period. Positioning-related and bleeding complications were relatively rare. It does not appear that intra or postoperative complications following urethral reconstructive surgery significantly impact the chance of eventual stricture recurrence at intermediate follow-up. 


\section{CONFLICT OF INTEREST}

None declared.

\section{REFERENCES}

1. Andrich DE, Dunglison N, Greenwell TJ, Mundy AR: The long-term results of urethroplasty. J Urol. 2003; 170: 90-2.

2. Andrich DE, Mundy AR: Urethral strictures and their surgical treatment. BJU Int. 2000; 86: 571-80.

3. Barbagli G, Palminteri E, Bartoletti R, Selli C, Rizzo M: Long-term results of anterior and posterior urethroplasty with actuarial evaluation of the success rates. J Urol. 1997; 158: 1380-2.

4. Santucci RA, Mario LA, McAninch JW: Anastomotic urethroplasty for bulbar urethral stricture: analysis of 168 patients. J Urol. 2002; 167: 1715-9.

5. Al-Qudah HS, Santucci RA: Extended complications of urethroplasty. Int Braz J Urol. 2005; 31: 315-23; discussion 324-5.

6. Rourke KF, Jordan GH: Primary urethral reconstruction: the cost minimized approach to the bulbous urethral stricture. J Urol. 2005; 173: 1206-10.

7. Wright JL, Wessells H, Nathens AB, Hollingworth W: What is the most cost-effective treatment for 1 to 2 $\mathrm{cm}$ bulbar urethral strictures: societal approach using decision analysis. Urology. 2006; 67: 889-93.

8. Erickson BA, Wysock JS, McVary KT, Gonzalez CM: Erectile function, sexual drive, and ejaculatory function after reconstructive surgery for anterior urethral stricture disease. BJU Int. 2007; 99: 607-11.
9. Bildsten SA, Dmochowski RR, Spindel MR, Auman JR: The risk of rhabdomyolysis and acute renal failure with the patient in the exaggerated lithotomy position. J Urol. 1994; 152: 1970-2.

10. Moses TA, Kreder KJ, Thrasher JB: Compartment syndrome: an unusual complication of the lithotomy position. Urology. 1994; 43: 746-7.

11. Anema JG, Morey AF, McAninch JW, Mario LA, Wessells H: Complications related to the high lithotomy position during urethral reconstruction. J Urol. 2000; 164: 360-3.

12. Angermeier KW, Jordan GH: Complications of the exaggerated lithotomy position: a review of 177 cases. J Urol. 1994; 151: 866-8.

13. Audry G, Johanet S, Achrafi H, Lupold M, Gruner M: The risk of wound infection after inguinal incision in pediatric outpatient surgery. Eur J Pediatr Surg. 1994; 4: 87-9.

14. Jakse G, Manegold E, Reineke T, Borchers H, Brehmer B, Wolff JM, et al.: Expanded, radical perineal prostatectomy. Urologe A. 2000; 39: 455-62.

15. Krieger JN, Bailey RC, Opeya J, Ayieko B, Opiyo F, Agot K, et al.: Adult male circumcision: results of a standardized procedure in Kisumu District, Kenya. BJU Int. 2005; 96: 1109-13.

16. Barbagli G, Palminteri E, Lazzeri M, Guazzoni G, Turini D: Long-term outcome of urethroplasty after failed urethrotomy versus primary repair. J Urol. 2001; 165: 1918-9.

17. Wood DN, Andrich DE, Greenwell TJ, Mundy AR: Standing the test of time: the long-term results of urethroplasty. World J Urol. 2006; 24: 250-4.

\section{Correspondence address:}

Dr. Chris M. Gonzalez

Department of Urology

675 North Saint Clair Street, Suite 20-150

Chicago, IL 60611

Fax: + 1312 695-7030

E-mail: cgonzalez@nmff.org 


\section{EDITORIAL COMMENT}

The authors reported on intra and postoperative complications of reconstructive urethral surgery performed by a single surgeon. Data consisted of 153 procedures performed on 128 patients including a variety of techniques, as well as use of grafts and flaps. The authors included in the series one stage and two-stage repairs.

The authors stated that their "study was a retrospective review of all urethral reconstructions over a 6 year span and their goal was to provide descriptive data from a large single institution experience which stratifies complications after various reconstructive procedures and their impact on stricture recurrence". My contribution as a reviewer is to wonder about today's impact on knowledge of heterogeneous single-institution series like this. Notice for instance that only 4 of 9 clinical subgroups listed included more than 20 patients on it. If the authors had limited their evaluation to only those series of patients they

\section{EDITORIAL COMMENT}

The take-home message from the manuscript by Navai et al. is that urethroplasty can be accomplished with high success rates and few complications. The authors also ask several important questions that seek to better understand the factors associated with complications after urethroplasty. Yet, there are several issues that make these questions difficult to answer. First, many of the variables examined by the authors and others in similar manuscripts are correlated with one another. For instance, the etiology of stricture (e.g. history of hypospadias repair) can be linked with the location of stricture (e.g. penile urethra), the length of stricture, type of repair (e.g. two-stage repair with buccal graft), patient positioning and operative time. Hence, to really understand what factors predict complications, these covariates would be better examined in a multiple logistic regression model. Second, most urethroplasty series are small as the disease is not common and is frequently managed by other means. This combined with the fact that the could probably achieve more conclusive results even though still not statistically significant.

We accept the authors' comment that "in the arena of urethral reconstruction, single surgeon series of enough volume to draw statistical conclusion are very difficult to come by". On the other hand, only if patients with urethral stricture are classified according to clinical characteristics and type of surgery we can truly rely on results and use this data to counsel our patients before surgery.

In conclusion the authors deserve merit for their paper but we acknowledge the need of cooperative studies to better evaluate the role of different urethral surgeries in regards to overall success and complications.

\section{Dr. Antonio Macedo Jr. Federal University of São Paulo Sao Paulo, Brazil E-mail:amcdjr@uol.com.br}

outcome of interest, in this case complications, is also rare makes it difficult to then do subset analyses to understand the predictors of the outcome. As the authors suggest, we will be better prepared to explore these issues when we approach them through a multiinstitutional collaborative database.

Still, the important lesson here remains that re-stenosis and complications are infrequent after urethroplasty. It remains the gold standard for the management of urethral stricture disease. The number of centers where patients can receive excellent definitive care for their urethral stricture continues to grow, as evidenced by Dr. Gonzales's experience.

Dr. Sean P. Elliott Department of Urologic Surgery University of Minnesota Minneapolis, Minnesota, USA E-mail: selliott@umn.edu 